\title{
CD3 Complex
}

National Cancer Institute

\section{Source}

National Cancer Institute. CD3 Complex. NCI Thesaurus. Code C38897.

Consisting of cell surface type I membrane Delta, Epsilon, Gamma, Zeta, and Eta protein subunits with ITAM domains and noncovalently associated with the disulfide bound heterodimeric alpha/beta and gamma/delta TCR, the CD3 complex couples receptor antigen recognition to signal transduction pathways during T-cell activation. During TCR eng agement with MHC-associated antigen on host cell surfaces and synapse formation, CD3 activity leads to Tyr-phosphorylated CD3 subunits, Tyr phosphorylation of LAT colocalized in lipid rafts, and MAPK activation. CD3 signal transduction appears to involve LCK, ZAP70, Nck, SLA, SLA2, and DOCK2. CD3 subunits may also associate with the cytoskeleton. CD3 also mediates TCR signal transduction during the developmental transition through positive selection of immature thymocytes to mature CD4+ or CD8+ T cells. $(\mathrm{NCl})$ 